\title{
Applications Research of Digital Technology in Printmaking Creation
}

\author{
Changhuan Chen
}

Guangzhou Academy of Fine Arts, Guangzhou, 510006, China

Keywords: Digital technology, Printmaking creation, Digital printmaking

\begin{abstract}
In recent years, the rapid development of digital technology is gradually applied to various fields of traditional art. Digital printmaking is an important achievement of the application of digital technology in printmaking creation. It enriches the concept of traditional printmaking and also brings great impact to traditional printmaking creation, which has the advantages of simplified production process, intense visual effect and broad woodcut scale. As an important artistic form of expression, digital printmaking will continue to be recognized and pursued by print artists and has good prospects for development.
\end{abstract}

\section{Introduction}

With the wide application of digital technology, human beings have entered the digital era. In this context, printmaking art also keeps pace with the times, which combines with digital technology, producing digital printmaking [1]. The emergence of digital printmaking has greatly broadened the artistic space of printmaking creation. Digital printmaking can create many works which only exist in the imagination of traditional printmakers. The emergence of digital printmaking is both an opportunity and a challenge for traditional printmaking. Only by actively absorbing the advanced technology and ideas in digital printmaking and combining the advantages of traditional printmaking itself, can traditional printmaking keep up with the trend of the times. Digital printmaking is a new product of the combination of traditional printmaking and today's digital technology. Because digital printmaking is created by digital technology, the works created by digital printmaking have the advantage of high accuracy and short time. At the same time, compared with traditional printmaking, digital printmaking in contemporary art is much more advanced in technology and material. Compared with the traditional printmaking production process, the digital printmaking production process has undergone tremendous changes in every part of the process. Traditional handcrafting has become the digital technology in contemporary art, traditional carving knives have become laser carving in contemporary art, and traditional printmaking and printing machines have become computer spraying in contemporary art. From the high-tech level, we positioned digital printmaking as the use of high-tech digital technology in traditional printmaking modeling language processed by computer technology and converted into material ink points, and then printed through the output platform with a limited number of complex printmaking. Digital printmaking is not only the inevitable product of digital technology to promote the progress of traditional printmaking art in the information age, but also the result of the innovation of digital technology in printmaking art. It enriches the concept of traditional printmaking. At the same time, it will bring infinite new development space for traditional prints [2].

\section{Creation Points of Digital Printmaking}

\subsection{Tools Selection.}

The comprehensive application of digital technology in digital printmaking has changed the printmaking from manual to digital [3]. We use computer software to process and extract images to complete plate-making work. There is mainly traditional manual combined with output plate-making, directly through the computer image processing software Photoshop or hand-drawn board to draw 
and create, using digital photographs, using four-color reduction method to produce film printing. The production of digital prints needs the help of professional image processing software Photoshop to complete the corresponding design and image processing. Photoshop software is a very powerful image processing software for pixel image processing. Digital printmaking also needs to be used in Corelraw, Painter, 3dmax and other software, because the creation process is a closely coordinated process, which cannot be separated from the assistance of these digital tools. Painter is a very important tool in painting. It constitutes a variety of functions of painting. It can quickly and effectively handle the elements needed in painting, give full play to the advantages of painting function in digital software, and bring unprecedented production experience. Chemical painting gets rid of the limitation of painting tools, promotes the development and innovation of the whole digital painting process, and brings far-reaching influence and significance. The emergence of digital printmaking is a new beginning of using new technology on the basis of traditional printmaking. It combines many elements of printmaking form and conforms to the formal characteristics of modern printmaking. The combination of digital technology and printing technology has led to the emergence of digital printmaking art. Any form of art appears in the language performance at the same time must grasp the performance of its tools, digital printmaking technology operation has a certain software familiarity and control tool learning process.

\subsection{Methods Application.}

There are behavioral and spatial differences between digital printmaking and traditional printmaking. Traditional printmaking is a conversational activity on the physical level with physical touch and temperature, while digital printmaking is a relatively virtual and untouchable digital behavior process. The influence of digital technology on modern art is also reflected in that it not only changes the traditional aesthetics, but also changes the artist's creative thinking. It makes the interaction between art and viewers possible. Art appreciation is no longer a one-sided view. In today's society, the concept of art has been popularized with the development of information technology in society, and the network and television have become the media of art communication. In general, there are two main types of digital printmaking. One is that in black-and-white woodcut, special treatment is carried out by means of digital tools, showing a contrast between tradition and modernity. The other is the digital printmaking made entirely by computer, through further screening of the alternative pictures by computer, editing and modifying them by digital software, integrating the traditional printmaking language into it, and finally using the printing material output. The first is digital original printmaking, which uses digital technology to transform the traditional printmaking into ink dots in the computer, and then through digital inkjet printing output has a certain amount of complex original printmaking. This is a new and controversial type of printmaking. But many international printmaking exhibitions have accepted this kind of digital printmaking and awarded awards to excellent digital printmaking. The second is digital reproduction printmaking, which relies on the modern high-tech stereo scanner with more than one billion pixels, scans the original works under authorization and supervision of the author or collector, and reproduces the original printmaking with high simulation degree through artistic micro-spray. The unique creative way of digital printmaking enables creators to use digital technology to carry out various forms of image processing experiments on different material media.

\subsection{Medias Output.}

In the output, we should convert the color design of Photoshop software into CMYK model to ensure its clarity [4]. Although printmaking has been completed in the computer, it is necessary to set printing parameters in the computer and output them. Generally speaking, the general printer can satisfy the output demand of printing. For small manuscripts and initial manuscripts, the general printer can be selected for processing. For high color requirements or final products, professional printers are needed to ensure the printing effect. Resolution adjustment is a very important part of printing. Before printing, we must adjust the resolution, improve the clarity and color effect of prints, check the whole printing effect comprehensively, and strive to achieve the best printing effect 
through various technical processing. We need to check the final effect of the screen. The output of media is of great significance to digital printmaking. It is the last link of digital printmaking and plays a very critical role. The most important equipment is the computer and digital inkjet printer, supplemented by scanners, hand-drawn boards, digital cameras and other equipment or accessories. In the aspect of creation, drawing software is mainly used for creation. The creation is mainly completed by computer. Printing works are output by digital inkjet printer. Nowadays, painters mostly choose high-quality "artistic micro-jet" printing mode. This process, called artistic micro-jet, requires artists to personally participate in the selection of materials such as ink, paper or canvas, to polish and correct their works, and even to personally operate high-quality large-scale inkjet printers to output their works. In fact, the process of artist's participation belongs to the creative process, during which there are often new inspirations and discoveries.

\section{Artistic Characteristics of Digital Printmaking}

\subsection{Simplified Production Process.}

Digital printmaking has unique advantages in simplifying the process of printmaking creation. Traditional printmaking is complicated and cumbersome. It needs to be divided into many different editions and printed on the same medium several times or dozens of times in a certain process. It requires a lot of physical strength and material workers. It is totally operated by bare hands, time-consuming, inefficient and even fruitless. Inspiration can sometimes be lost in complicated mechanical work. This is the greatest loss and distress of artists. Digital printmaking is different from traditional printmaking. Digital printmaking is a process of making plate in the computer, which is made by dozens or hundreds of times of overlapping. Any program can be modified in the process of making. This greatly saves the energy consumed by traditional prints, and ultimately results in a complete printmaking. In the production of digital printmaking, you can use a variety of graphic painting software to complete the work at one time. As long as you master certain computer image software technology, you can achieve many effects by using filters and other features. More importantly, digital printmaking can be revised repeatedly in the production process, avoiding accidental mistakes and technical errors in the creation. As long as the creator is proficient in the use of software, he can be handy.

\subsection{Intense Visual Effect.}

As a product of digital media, digital printmaking has virtual power. Therefore, some special effects can be achieved by virtue of digital image virtual technique. Because its carrier is different from the previous media, its appearance is also different, the artistic space and field of expression is also extensive and profound. We should understand the special aesthetic feeling and new visual effect brought about by digital printmaking and digital era from the ideological point of view. Digital technology enriches creative thinking and brings them broader creative inspiration. Because of the limitations of traditional printmaking techniques, the picture effect has not broken through the conventional. Digital printmaking is rich and intense, free and unrestrained. The color matching of computer image software extends the expression space of color. It is intuitive and convenient to select color on existing color panels. And the digital works printed by laser or inkjet printer are exactly the same as the original design in color restoration. This will undoubtedly add infinite help to the creators with weak color ability and provide more space for artists. In the process of creating digital prints, there will be many contingencies and randomness in the picture effect after computer software processing. This characteristic makes digital prints have a great space of expression and innovation. In addition to synthesizing the expressive skills of various types of prints, it can also simulate the expressive forms and techniques of traditional prints, and produce more rich effects that traditional prints cannot express, so that the pictures show different inclusiveness and diversity. 


\subsection{Broad Woodcut Scale.}

In the traditional printmaking creation, the size of the works is mostly small, and it is very difficult to make large-scale printmaking works. Printmaking works depend on machine rolling printing. Due to space and cost constraints, schools or individual studios are mainly small and medium-sized printmaking machines, and the size of the work must be smaller than the size of the printmaking machine press. Large-scale works have a high demand on the physical strength, energy and skills of artistic creators. The bigger the picture, the more difficult it is to control the shape, the more details are reflected, and the richer the effect of the picture is. Digital printmaking has no requirement for the size of the material. It can enlarge or shrink the picture arbitrarily without any influence on the accuracy of the work.

\section{Development Prospect of Digital Printmaking}

The close combination of science and technology with art is an innovative approach and development trend in the field of art. Traditional prints are mostly presented in the form of plane, or as a whole or cut into small pieces to display, the form is flat and single. How to attract the audience's eyes with novel display methods has also become a part of the creation. Breakthrough the traditional two-dimensional plane display form to three-dimensional picture transition, printmaking language from simple plane image to dynamic image is the promotion of digital printmaking display space. Digital printmaking has developed rapidly in a few years since it was introduced into China from abroad. In the rapid social change, digital printmaking conforms to the development trend of the times, conforms to the rich and changeable aesthetic standards of the information age, and wins a vast living space in the contemporary era with its unique accuracy, conciseness and high efficiency. The emergence of digital printmaking has brought new ideas for the development of printmaking art. It has thoroughly broken the limitations of traditional printmaking in production techniques, better catered to the trend of diversified development of information in modern society, and gradually liberalized and diversified the concept of contemporary printmaking art. In the form of expression, digital printmaking integrates the characteristics of traditional printmaking art with modern artistic elements to make it look new. It can be foreseen that in the future development of digital printmaking, there will be more and more creative phenomena with comprehensive media materials and cross-fields. With the development of information technology, the frequency of human information communication and communication is explosively increasing. Skillful technology and systematic logical thinking ability are the necessary conditions for the success of digital printmaking. As long as the tools and manipulations in image design software are changed into a different collocation method and order, the same graphics and images will produce completely different effects. With the improvement of people's material living standard, people's pursuit of spiritual life will be higher and higher. As an elegant and important artistic expression form, digital printmaking will continue to be recognized and pursued by people. As a pioneer of cross-border art, digital printmaking will certainly have a broader development prospect and a deeper space for artistic expression.

\section{Conclusion}

The rapid development of science and technology has promoted the continuous progress of the whole human society, brought opportunities and challenges to artistic creation. The emergence of digital technology has broadened the artist's vision, enriched the content of artistic creation, stimulated the inspiration of artist's creation and promoted the development of artistic creation. Digital printmaking is the inevitable product of the development in the digital era. Digital printmaking is a brand-new art form which is produced by the combination of technology and art. In the future development, digital printmaking will give full play to the creativity and artistic expression of art on the basis of advanced science and technology to enhance the aesthetic connotation of digital printmaking in the integration of technology and art. 


\section{References}

[1] Guo Jianwen. The Technique without Print Trace, The Non-Mechanical Character of Digital Print [J]. Art and Design, 2015(6): 121-122.

[2] Lv Qing. On the Form Creation of Digital Engraving in Contemporary Art [J]. Education Teaching Forum, 2018(48): 46-47.

[3] Li Chuanyang. The Application and Reflection of Digital Technology in Contemporary Print Art [J]. The Science Education Article Collects, 2012(7): 204-205.

[4] Zhang Yamin. Affinity and Artistic Representation of Silk Screen Art [J]. Design Research, 2016, 6(6): 34-38. 